УДК $517.53+517.55$

\title{
A Refinement of Kovalevskaya's Theorem on Analytic Solvability of the Cauchy Problem
}

\author{
Alexander A. Znamenskiy* \\ Institute of Mathematics and Computer Science \\ Siberian Federal University \\ Svobodny, 79, Krasnoyarsk, 660041
}

Russia

Received 25.11.2016, received in revised form 20.05.2017, accepted 10.07.2017

In this paper we give a proof of an analog of the Kovalevskaya theorem about analytic solvability of the Cauchy problem for a linear differential equation with constant coefficients. A major role in the proof is played by the Borel transform and the Laurent expansion of the function $P^{-1}$, where $P$ is the characteristic polynomial. This expansion produces an efficiently computable approximation of the solution of the Cauchy problem. The method of the proof allows to consider equations not necessarily resolved with respect to the highest derivative, however it imposes additional restrictions on the right hand side.

Keywords: Cauchy problem, Borel transform, Newton polytope, Laurent expansion.

DOI: $10.17516 / 1997-1397-2017-10-4-531-536$.

\section{Introduction}

Consider a Cauchy problem for a linear partial differential equation. In the traditional formulation of the Cauchy-Kovalevskaya theorem it is assumed that the equation is resolved with respect to the pure (not mixed) derivative of the highest order, for example, with respect to $\partial^{m} / \partial x_{n}^{m}$, where $m$ is the order of the differential equation. Namely, the equations considered are of the form

$$
\frac{\partial^{m} y}{\partial x_{n}^{m}}=\sum_{|\alpha| \leqslant m}^{\prime} a_{\alpha}(x) \mathcal{D}^{\alpha} y+f
$$

where

$$
a_{\alpha}(x)=a_{\alpha_{1} \ldots \alpha_{n}}\left(x_{1}, \ldots, x_{n}\right), \quad \mathcal{D}^{\alpha} y=\frac{\partial^{\alpha_{1} \cdot \ldots \cdot \alpha_{n}} y}{\partial x_{1}^{\alpha_{1}} \cdot \ldots \cdot \partial x_{n}^{\alpha_{n}}},
$$

and the summation is taken over derivatives of orders $|\alpha|:=\alpha_{1}+\cdots+\alpha_{n} \leqslant m$, except for $\frac{\partial^{m} y}{\partial x_{n}^{m}}$. In this case the initial data is usually the following

$$
\frac{\partial^{k} y}{\partial x^{k}}\left(x^{\prime}, 0\right)=y_{k}\left(x^{\prime}\right), \quad k=0, \ldots, m-1
$$

where $x^{\prime}=\left(x_{1}, \ldots, x_{n-1}\right)$.

Kovalevskaya proved [1] that for any analytic in a neighborhood of the origin coefficients, $a_{\alpha}(x)$, function $f(x)$, and initial data (2) the problem (1), (2) has a unique analytic solution $y(x)$.

In the case of constant coefficients we consider a more general equation than (1). Namely, let $P$ be a polynomial

$$
P=z_{n}^{m}+\sum_{\alpha \in A} a_{\alpha} z^{\alpha}
$$

*msznam@gmail.com

(C) Siberian Federal University. All rights reserved 
where $A \subset \mathbb{Z}_{\geqslant 0}^{n-1} \times\{0,1, \ldots, m-1\}$ is a fixed finite set of exponents. Then $P$ as a characteristic polynomial defines a differential operator $P(\mathcal{D})$; we consider a differential equation

$$
P(\mathcal{D}) y=f
$$

with right hand side $f=\sum_{k \in \mathbb{Z}_{\geqslant 0}^{n}} b_{k} x^{k}$ given by a power series.

Note that the leading monomial of $P$ with respect to variable $z_{n}$ has the form $z_{n}^{m}$, the degree of $z_{n}$ in all other monomials is less than $m$, degrees of the remaining variables are arbitrary. For equation (4) this implies that it contains the derivative $\frac{\partial^{m}}{\partial x_{n}^{m}}$, which is the highest with respect to $x_{n}$, but not necessarily the highest derivative in the equation. Then the Cauchy problem for equation (4) consists in finding a solution $y(x)$ of (4) with initial data of the form (2).

Note that we can always assume that the initial data vanish making the substitution $y=\tilde{y}+\phi$, where

$$
\phi=\sum_{j=0}^{m-1} \frac{x_{n}^{j} y_{j}\left(x^{\prime}\right)}{j !} .
$$

Then after substitution of $\tilde{y}+\phi$ instead of $y$ in the $k$-th equation of the system $(2), k=$ $0,1, \ldots, m-1$ we get the equality

$$
\frac{\partial^{k}}{\partial x_{n}^{k}} \tilde{y}+\frac{\partial^{k}}{\partial x_{n}^{k}} \sum_{j=0}^{m-1} \frac{x_{n}^{j} y_{j}\left(x^{\prime}\right)}{j !}=y_{k}\left(x^{\prime}\right) .
$$

After differentiation of the sum $\sum_{j=0}^{m-1} \frac{x_{n}^{j} y_{j}\left(x^{\prime}\right)}{j !}$ the first $k-1$ summands will be zeroes, the $k$-th summand will become $y_{k}\left(x^{\prime}\right)$, and the rest will vanish since they contain the vanishing factor $x_{n}$. Moving $y_{k}\left(x^{\prime}\right)$ to the left hand side of the equality and simplifying the expression, we get zero initial data

$$
\frac{\partial^{k}}{\partial x_{n}^{k}} \tilde{y}=0, \quad k=0,1, \ldots, m-1 .
$$

Since this transformation does not change the form of the equation, without loss of generality we assume that we are given zero initial data from the beginning.

Now we can formulate the theorem that will be proved in the rest of the paper.

Theorem 1. If the right hand side $f$ of equation (4) is an entire function of exponential type then the Cauchy problem (4), (2) has a unique analytic solution.

The severe restriction on the right hand side $f$ of the equation is dictated by the Borel transform used in the proof. The condition of $f$ being an entire function of exponential type ensures that the Borel transform of $f$ has a non-empty domain of convergence.

Note that for an arbitrary support $A$ of summation in (3) the restrictions on the right hand side of the equation become essential, which is demonstrated by the well-known example of Kovalevskaya for the heat equation ([1], p. 22).

Thus, a more general form of the equation implies stricter conditions on the right hand side. And vice versa, if an equation is in the generalized Kovalevskaya class then, as Korobeinik showed [2], the existence of the solution is established even for more general classes of functions than analytic.

Note that in relation to equations with constant coefficients of the form (1) the Borel transform has been employed in [3] to obtain an integral representation for a solution of the corresponding Cauchy problem. 


\section{The Borel transform and properties of the characteristic polynomial}

Definition 1. Let the function

$$
f(x)=\sum_{k \in \mathbb{Z}_{\geqslant 0}^{n}} b_{k} x^{k}
$$

be analytic in a neighborhood of the origin. The function

$$
B_{f}(z)=\sum_{k \in \mathbb{Z}_{\geqslant 0}^{n}} \frac{b_{k} \cdot k !}{z^{k+I}}
$$

where $I$ is the unit multi-index, is called the Borel transform of $f$ [4].

Let us assume that the Taylor coefficients $b_{k}$ of $f$ decrease rapidly to ensure that the domain of convergense of the series $B_{f}$ is not empty. For example, entire functions of exponential type have this property. By Abel's lemma such a domain of convergence must contain the set $\left|z_{1}\right| \geqslant$ $R_{1}, \ldots,\left|z_{n}\right| \geqslant R_{n}$. Therefore the series converges uniformly on the torus $\left|z_{1}\right|=R_{1}, \ldots,\left|z_{n}\right|=R_{n}$, and by term-wise integration we get

$$
\frac{1}{(2 \pi i)^{n}} \int_{\left|z_{1}\right|=R_{1}} \ldots \int_{\left|z_{n}\right|=R_{n}} B_{f}(z) e^{\langle z, x\rangle} d z_{1} \wedge \ldots \wedge d z_{n}=f(x) .
$$

Let $\Delta$ be a polytope, i.e. the convex hull of a finite set of points in $\mathbb{R}^{n}$.

Definition 2. The dual cone of the polytope $\Delta$ at the point $p \in \Delta$ is the set

$$
C_{p}=C_{p}(\Delta)=\left\{q \in\left(\mathbb{R}^{n}\right)^{*}: \max _{\alpha \in \Delta}\langle q, \alpha\rangle=\langle q, p\rangle\right\} .
$$

Thus, the dual cone $C_{p}$ consists of all functionals $q$ whose maximal values on $\Delta$ are attained at the point $p[5]$.

Definition 3. Let $P=\sum_{\alpha \in A} a_{\alpha} x^{\alpha}$ be a polynomial. The Newton polytope $\Delta_{P}$ of $P$ is the convex hull in $\mathbb{R}^{n}$ of the set $A \subset \mathbb{Z}^{n} \subset \mathbb{R}^{n}$ of exponents $\alpha$ such that $a_{\alpha}$ is not zero.

By $\stackrel{\circ}{C}$ we shall denote the interior of the set $C \in \mathbb{R}^{n}$.

Lemma 1. Let $v$ be a vertex of the Newton polytope $\Delta_{P}$ of the polynomial $P$, and $C_{v}$ be the dual cone of $\Delta_{P}$ at the vertex $v$. Then for any $c \in C_{v}$ the torus

$$
T_{\rho}=\left\{\left|z_{1}\right|=\rho^{c_{1}}, \ldots,\left|z_{n}\right|=\rho^{c_{n}}\right\}
$$

does not intersect the hypersurface $V=\{z: P(z)=0\}$ for $\rho \gg 1$.

Proof. Let $P(z)=\sum_{\alpha \in A} a_{\alpha} z^{\alpha}$. The restriction of $P(z)$ on $T_{\rho}$ is

$$
\left.P\right|_{T_{\rho}}=\sum_{\alpha \in A} a_{\alpha} e^{i\langle\theta, \alpha\rangle} \rho^{\langle c, \alpha\rangle}
$$

Since $c \in \stackrel{\circ}{C}_{v}$ and $v$ is a vertex of $\Delta_{P}$, the maximum on $\Delta_{P}$ of the scalar product $\langle c, \alpha\rangle$ is attained at the only point $v$. This means that for sufficiently big $\rho$ the monomial

$$
\left(a_{v} e^{i\langle\theta, v\rangle}\right) \rho^{\langle a, v\rangle}
$$

dominates in absolute value the remaining monomials as well as their sum. Therefore, the restriction (8) does not vanish for $\rho \gg 1$. 
Let now $P$ be a polynomial of the form (3). It is obvious that the point $v=(0, \ldots, 0, m)$ is a vertex of $\Delta_{P}$.

Lemma 2. For $v=(0, \ldots, 0, m)$ there exists $c \in \stackrel{\circ}{C}_{v}$ such that for sufficiently big $\rho \gg 1$ the torus $T_{\rho}$ lies in the domain of convergence of the series $B_{f}$ and does not intersect the hypersurface $\{z: P(z)=0\}$.

Proof. Let us show that the dual cone $\dot{C}_{v}\left(\Delta_{P}\right)$ at the vertex $v=(0, \ldots, 0, m)$ contains a vector $c=\left(c_{1}, \ldots, c_{n}\right)$ with positive coordinates. The vector $c$ we shall find in the form $(\varepsilon, \ldots, \varepsilon, 1)$, where $\varepsilon$ is real positive.

The fact of $c$ lying in the interior $\dot{C}_{v}\left(\Delta_{P}\right)$ is expressed by the condition that the maximum

$$
\max _{\alpha \in \Delta_{P}}\langle c, \alpha\rangle
$$

is attained at the only point $\alpha=v$.

The value of the function $\langle c, \alpha\rangle$ for $\alpha=v$ is equal to $m$. For all other points $\alpha \in \Delta_{P}$

$$
\langle c, \alpha\rangle=\left(\varepsilon\left(\alpha_{1}+\ldots+\alpha_{n-1}\right)+\alpha_{n}\right),
$$

and $\alpha_{n}<m$. Since the function $\alpha_{1}+\ldots+\alpha_{n-1}$ is bounded on the compact set $\Delta_{P}$, the maximal value of (10) is less than $m$ provided $\varepsilon$ is sufficiently small. Thus, the existence of $c$ is proved.

Now by Lemma 1 , for $\rho \gg 1$ the torus $T_{\rho}=\left\{\left|z_{1}\right|=\rho^{c_{1}}, \ldots,\left|z_{n}\right|=\rho^{c_{n}}\right\}$ does not meet the zero set of the polynomial $P(z)$. For sufficiently large $\rho$ the torus $T_{\rho}$ lies within the domain convergence of the series $B_{f}$.

\section{The proof of Theorem 1}

Introduce the following integral with parameter $x$ :

$$
y(x)=\frac{1}{(2 \pi i)^{n}} \int_{\left|z_{1}\right|=R_{1}} \ldots \int_{\left|z_{n}\right|=R_{n}} \frac{B_{f}(z) e^{\langle z, x\rangle}}{P(z)} d z_{1} \wedge \ldots \wedge d z_{n}:=\oint \frac{B_{f}(z) e^{\langle z, x\rangle}}{P(z)} d z .
$$

Note that for $R_{j}=\rho^{c_{j}}$, where $\rho \gg 1$, this integral is well-defined: according to Lemma 2 in this case the set of integration does not intersect the zeroes of the integrand's denominator.

Differentiating the integral, we see that (11) satisfies equation (4):

$$
P(\mathcal{D}) y(x)=\oint \frac{B_{f}(z) P(\mathcal{D}) e^{\langle z, x\rangle}}{P(z)} d z=\oint B_{f}(z) e^{\langle z, x\rangle} d z=f(x) .
$$

Here we use the equality $P(\mathcal{D}) e^{\langle z, x\rangle}=P(z) e^{\langle z, x\rangle}$.

Represent the polynomial $P(z)$ as $P(z)=z_{n}^{m}-\theta(z)$. Then

$$
\frac{1}{P(z)}=\frac{1}{z_{n}^{m}-\theta(z)}=\frac{1}{z_{n}^{m}\left(1-\frac{\theta(z)}{z_{n}^{m}}\right)}=\frac{1}{z_{n}^{m}} \sum_{l=0}^{\infty}\left(\frac{\theta(z)}{z_{n}^{m}}\right)^{l}
$$

this series converges since the monomial $z_{n}^{m}$ on $T_{\rho}$ dominates in absolute value the sum of the remaining monomials, as follows from the proof of Lemma 1. It follows that the integral (11) is given by the following series

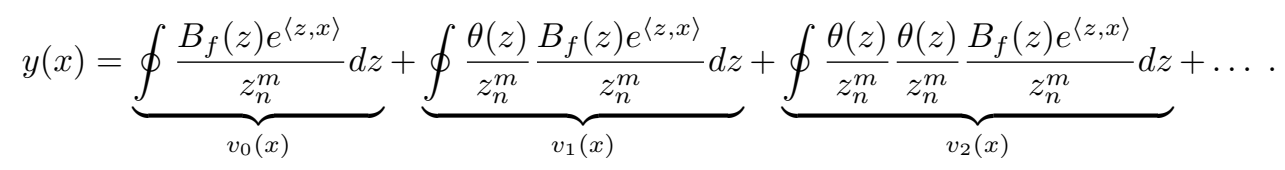


Let us show that the solution $y(x)$ satisfies homogeneous initial data (6). In order to do this it is enough to show that each term of the sequence $v_{\nu}$ satisfies the conditions (6). We have

$$
\begin{aligned}
\frac{\partial^{s}}{\partial z_{n}^{s}} v_{\nu}\left(x^{\prime}, 0\right)=\oint\left(\frac{\theta(z)}{z_{n}^{m}}\right)^{\nu} \frac{B_{f}(z) e^{\left\langle z^{\prime}, x^{\prime}\right\rangle}}{z_{n}^{m-s}} & d z= \\
& =\oint\left(\frac{\theta(z)}{z_{n}^{m}}\right)^{\nu} e^{\left\langle z^{\prime}, x^{\prime}\right\rangle} \sum_{k \in \mathbb{Z}_{\geqslant 0}^{n}} \frac{b_{k} \cdot k !}{\left(z^{\prime}\right)^{k^{\prime}+I^{\prime}} \cdot z_{n}^{k+1+m-s}} d z .
\end{aligned}
$$

Here the degree of $z_{n}$ in each summand $\frac{b_{k} \cdot k !}{\left(z^{\prime}\right)^{k^{\prime}+I^{\prime}} \cdot z_{n}^{k+1+m-s}}$ does not exceed -2 , since $s<m$, $k \geqslant 0$ and therefore $k+1+m-s \geqslant 2$. The degree of $z_{n}$ in the expression $\left(\frac{\theta(z)}{z_{n}^{m}}\right)^{\nu}$ can not be positive. Taking into account that

$$
\oint \frac{d z}{z^{j}}= \begin{cases}1, & \text { if } j=I \\ 0, & \text { otherwise }\end{cases}
$$

we obtain

$$
\frac{\partial^{s}}{\partial z_{n}^{s}} v_{\nu}(0)=0, \quad m-1 \geqslant s \geqslant 0 .
$$

Now we construct a sequence of functions convergent to a solution of (4). Denote by $\mathcal{D}_{n}^{-m} f$ an antiderivative of $f$ of order $m$ with respect to variable $x_{n}$. Such antiderivatives are defined up to polynomials of degree $m-1$. For instance, if a function $f(x)$ is given by a series

$$
f(x)=\sum_{k \in \mathbb{Z}_{\geqslant 0}^{n}} b_{k} x^{k},
$$

then its antiderivative $\mathcal{D}_{n}^{-m} f(x)$ is given by the series

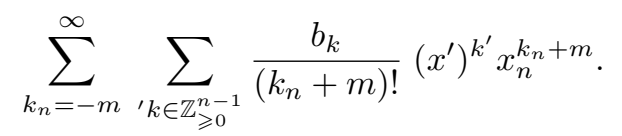

We shall only choose antiderivatives such that $b_{k}=0$ for all $k_{n}<0$. This implies that the solution satisfies the homogeneous initial data.

Thus, we have a sequence

$$
\begin{aligned}
& v_{0}(x)=\mathcal{D}_{n}^{-m} f, \\
& v_{1}(x)=\left(\mathcal{D}_{n}^{-m} \theta(\mathcal{D})\right) \mathcal{D}_{n}^{-m} f, \\
& \ldots \\
& v_{\nu}(x)=\left(\mathcal{D}_{n}^{-m} \theta(\mathcal{D})\right)^{\nu} \mathcal{D}_{n}^{-m} f, \\
& \ldots .
\end{aligned}
$$

For the partial sum $y_{\nu}(x)=\sum_{k=0}^{\nu} \nu_{k}(x)$ we obtain

$$
\mathcal{D}_{n}^{m} y_{\nu}(x)=\mathcal{D}_{n}^{m}\left(\mathcal{D}_{n}^{-m} f+\mathcal{D}_{n}^{-m} \theta \mathcal{D}_{n}^{-m} f+\cdots+\left(\mathcal{D}_{n}^{-m} \theta\right)^{\nu} \mathcal{D}_{n}^{-m} f\right)=f(x)+\theta(\mathcal{D}) y_{\nu-1}(x) .
$$

Passing to the limit as $\nu \rightarrow \infty$, we get $\mathcal{D}_{n}^{m} y(x)-\theta(\mathcal{D}) y(x)=f(x)$.

It is necessary to note that this iteration coincides with the iteration from a well-known theorem of Hörmander [6]. However its convergense is established there differently. Using this iteration we can consider (as a series (12)) equation (4) with variable coefficients (and even some non-linear equations). 


\section{References}

[1] Sophie von Kowalevsky, Zur Theorie der partiellen Differentialgleiehungen, Journal für Mathematik, 1874, Bd. LXXX. Heft 1.

[2] Yu.F.Korobeinik, Representative systems of exponentials and the Cauchy problem for partial differential equations with constant coefficients, Izvestiya: Mathematics, 61(1997), no. 3, $553-592$.

[3] E.Leinartas, The Cauchy problem in a class of entire functions in several variables, Banach Center Publications, 33(1996), no. 1, 189-192.

[4] L.I.Ronkin, Introduction to the theory of entire functions of several variables. Translations of Mathematical Monographs, 1974.

[5] A.G.Khovanskii, Newton polyhedra and toroidal varieties, Funct. Anal. Appl., 11(1977), no. 4, 289-296

[6] L.Hörmander, The Analysis of Linear Partial Differential Operators II. Differential Operators with Constant Coefficients, 2005.

\section{Одно уточнение теоремы Ковалевской об аналитической разрешимости задачи Коши}

Александр А. Знаменский

Институт математики и фундаментальной информатики Сибирский федеральный университет

Свободный, 79, Красноярск, 660041

Россия

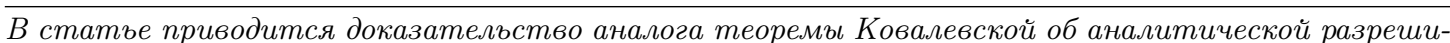
мости задачи Коши для линейного дифберенциального уравнения с постоянными коэфбициентами. В этом доказательстве важную роль играют преобразование Бореля и разложение Лорана функиии $P^{-1}$, где $P$ - характеристический многочлен. Такое разложение продуцирует рационально вычислимую аппроксимацию решения задачи Коши. Этот метод доказателъства позволяет рассматривать уравнения, не обязателъно разрешенные относителъно производной старшего порядка, однако накладьвает ограничение на правую часть уравнения.

Ключевые слова: задача Коши, преобразование Бореля, многогранник Нъютона, разложение Лорана. 